\title{
Systemic risk factors correlated with hyperhomocysteinemia for specific MTHFR C677T genotypes and sex in the Chinese population
}

\author{
Tianyuan Xiang ${ }^{1,2 \#}$, Hang Xiang ${ }^{2,3 \#}$, Muyang Yan ${ }^{4}$, Sheng $\mathrm{Yu}^{5}$, Matthew John Horwedel ${ }^{6}$, Yang $\mathrm{Li}^{3}$, \\ Qiang Zeng'
}

${ }^{1}$ China Health Management Institute, The Second Medical Center \& National Clinical Research Center for Geriatric Diseases, Chinese PLA General Hospital, Beijing, China; ${ }^{2}$ Eli and Edythe Broad CIRM Center for Regenerative Medicine and Stem Cell Research at USC, Los Angeles, CA, USA; ${ }^{3}$ Department of Cardiology, Chinese PLA General Hospital, Beijing, China; ${ }^{4}$ Department of Hyperbaric-Oxygen, Chinese PLA General Hospital, Beijing, China; ${ }^{5}$ Department of Hepatobiliary Surgery, Nanfang Hospital, Southern Medical University, Guangzhou, China; ${ }^{6}$ Division of Engineering in Medicine, Brigham and Women's Hospital, Harvard Medical School, Cambridge, MA, USA

Contributions: (I) Conception and design: Q Zeng, Y Li; (II) Administrative support: T Xiang, H Xiang; (III) Provision of study materials or patients: Q Zeng, T Xiang; (IV) Collection and assembly of data: M Yan, S Yu; (V) Data analysis and interpretation: T Xiang, MJ Horwedel; (VI) Manuscript writing: All authors; (VII) Final approval of manuscript: All authors.

\#These authors contributed equally to this article.

Correspondence to: Yang Li, MD, PhD. Department of Cardiology, the Second Medical Center, Chinese PLA General Hospital, Beijing, China. Email: liyangbsh@163.com; Qiang Zeng, MD, PhD. China Health Management Institute, The Second Medical Center \& National Clinical Research Center for Geriatric Diseases, Chinese PLA General Hospital, Beijing, China. Email: zq301@126.com.

Background: Methyltetrahydrofolate reductase (MTHFR) is a main regulatory enzyme in homocysteine (Hcy) metabolism. A common C677T mutation in the MTHFR gene results in decreased enzyme activity, which contributes to hyperhomocysteinemia (HHcy). Previous studies have shown that HHcy was correlated with various systemic diseases, such as cardiovascular disease, stroke, cancer, renal failure and so on. However, we hypothesized that HHcy in different genotype and sex groups may have different risk factors, which would lead to various pathologic states. Therefore, the aim of this study was to explore systemic information that are correlated with HHcy for specific MTHFR C677T genotypes and sex, which might be useful for predicting and preventing systemic diseases.

Methods: This cross-sectional study was performed through November 2017 to July 2019. A total of 4,534 adults aged 20-75 y were selected for this study. All the participants underwent a physical examination, blood tests and MTHFRC677T genotyping. Multivariable linear regression was performed to explore the risk factors for HHcy for each sex and genotype.

Results: The average of Hcy level is higher in the TT genotype than CC and CT genotypes $(\mathrm{P}=0.000)$. Multiple linear regression analysis identified the common protective factors (folate and Vit B12) and risk factor (Cr) for HHcy. Besides that, each group has its specific risk factors-female-CT (age, SBP, and Hb), female-TT (SBP and AST); male-CC (age, AST and Hb), male-CT (age and AST) and male-TT (SBP, AST, and $\mathrm{Hb}$ ).

Conclusions: HHcy was associated with different risk factors for each specific sex and genotype. These risk factors might be useful for predicting and preventing systemic diseases.

Keywords: Hyperhomocysteinemia; MTHFR polymorphism; sex; risk factor

Submitted Sep 01, 2020. Accepted for publication Nov 03, 2020.

doi: $10.21037 / \mathrm{atm}-20-6587$

View this article at: http://dx.doi.org/10.21037/atm-20-6587 


\section{Introduction}

The metabolism of Hcy is regulated by various enzymes and B vitamins (1). Elevated levels of Hcy can be caused by genetic mutation and enzyme deficiency. The enzyme of MTHFR is important for the folate metabolism which is an integral process for cell metabolism in the DNA, RNA and protein methylation (2). The mutation of the MTHFR C677T would reduce the activity of this enzyme, and predispose to HHcy under conditions of impaired folate status $(3,4)$. HHcy can also be caused by deficiencies in the folate, vitamin B12 and B6 vitamin (lesser extent) $(1,5)$. Furthermore, some physiologic and lifestyle determinants have also been identified. For example, increasing age, male sex, food intake, smoking, and heavy coffee consumption would increase Hcy concentration (6), whereas young age, premenopausal state, pregnancy, B vitamin intake, and exercise are associated with low Hcy concentrations (7).

High levels of Hcy, with or without MTHFR C677T polymorphism, is associated with various diseases (5), such as cardiovascular diseases(CVD) $(8,9)$, stroke (10), and the neural tube defects (10), in addition to chronic renal failure (11), ulcerative colitis (12), cancers (13-15), dyslipidemia (16), infertility (17), and so on $(18,19)$. Therefore, serum Hcy level becomes a potential therapeutic target for preventing or predicting systemic diseases (20).

We hypothesized that each sex and genotype might have specific risk factors associate with HHcy. For example, HHcy patients with MTHFR C677T homozygous genotype (TT) might have different risk factors from those with wild (CC)/heterozygous genotype (CT). In addition, some studies have shown an enhanced sex effect of Hcy concentration after puberty ( $>15 \mathrm{y}$ ), even though the difference was slight at an early age (21). Therefore, we also take sex into consideration, as each sex has different average of Hcy level and different spectrum of systemic diseases.

Thus, the aim of this study was to explore the systemic risk factors associated with HHcy in specific genotype and sex, which might be used for predicting or preventing systemic diseases.

We present the following article in accordance with the MDAR reporting checklist (available at http://dx.doi. org/10.21037/atm-20-6587).

\section{Methods}

\section{Study design and etbics approval}

The Ethics Committee at PLA General Hospital approved the protocol of this cross-sectional, observational, nonrandomized study (clinical trial ID: S2016-098-02), which was designed following the principle of the Declaration of Helsinki (as revised in 2013). All subjects gave their informed consent to take part in the study. The study was conducted at the Health Management Institute of Chinese PLA General Hospital from November 2017 to July 2019. Written informed consent was obtained from all patients to publish their information in nominate data.

\section{Subjects}

A total of 4,770 participants who completed a health examination were enrolled in this study. The inclusion criteria were: aged between 20 and 80 , not taking folic acid supplements or using agents that affect vitamin $B$ and folic acid metabolism, including methotrexate and anticonvulsants, and being free from folic medicine for at least 6 months.

Among those participants, 34 participants with severe renal disease, 54 participants with hepatic disease, and 123 participants with hypothyroidism were excluded from the study. Also, 25 participants were excluded from the inaccuracy of the results. In all, 1,369 females and 3,165 males were selected for studying in this crosssectional study.

\section{Outcome measures}

\section{Assessment of MTHFR C677T genotype}

Genetic polymorphisms MTHFR $677 \mathrm{C} \rightarrow \mathrm{T}$ were detected using gene chip hybrid analysis. Genomic DNA was extracted from the whole blood of the participants using the QIAamp ${ }^{\circledR}$ DNAMiniKit (CAT No. 51304, Germany). The PCR, hybridization, gene array detection, and analysis were conducted according to the manuals of the BaiO genotype detecting gene array kits and equipment (BaiO Technology Corp).

\section{Assessment of covariate}

The patients were subjected to a health examination, including height and weight measurements and blood pressure. Fasting blood was extracted for detection. HPLC analyzed plasma Hcy with fluorometric detection $(22,23)$. Folate concentration was measured using a dual count Solid Phase Boil Radio assay (Diagnostic Products, Los Angeles, CA). The vitamin (Vit) B12 was detected by liquid chromatography-tandem mass spectrometry (24). The blood 
glucose levels were measured using the hexokinase method, serum levels of bilirubin, Alanine aminotransferase (ALT), and Asparagine aminotransferase (AST) were measured using a BM Hitachi 711 Chemistry Analyzer. The total cholesterol (TC), high-density lipoprotein (HDL), lowdensity lipoprotein $(\mathrm{LDH})$, and TG levels were measured with a colorimetric method (Cobas c, 501 autoanalyzers, Roche Diagnostics, Germany). Serum Cr and UA were measured by a modified kinetic rate Jaffe reaction method using a Dade Dimension Chemistry Analyzer (Siemens). Cancer biomarkers of CEA and AFP are measured using electrochemiluminescence immunoassay. As the baseline of the Hcy level was varied between sexes, HHcy was defined distinctively for males $(\geq 15 \mu \mathrm{mol} / \mathrm{L})$ and female $(\geq 10 \mu \mathrm{mol} / \mathrm{L})$ $(25,26)$. Standard quality control procedures were performed each day with standard samples $(\mathrm{CV}<10 \%)$.

\section{Statistical analysis}

Sexes and genotypes grouped participants. A comparison of the variables was assessed using an ANOVA or Chi-square test. Multiple linear regression analyses were performed between Hcy and the variables with stepwise methods. The standard coefficients for the variables are used to estimate the strength of the association. All tests were two-tailed, and $P$ values less than 0.05 were considered to show significant differences. The statistical analyses were conducted using SPSS version 17.0 and SAS version 8.02 or 9.1 .

\section{Results}

\section{Clinical characteristics}

There were 4,534 individuals selected for this study, including 3,165 males ( $\mathrm{CC}=724$, CT $=1,523$ and $\mathrm{TT}=918$ ) and 1,369 females $(\mathrm{CC}=320, \mathrm{CT}=683$ and $\mathrm{TT}=366$ ). There were significant differences between males and females in the clinical characteristics (Table S1).

Further analysis was performed in each sex group and stratified by genotypes. For males, the Hcy level is significantly different between three genotypes with the mean values of $12.30 \pm 3.34,12.94 \pm 4.57$, and $19.37 \pm 9.26 \mu \mathrm{mol}$ for male-CC, male-CT, and male-TT, respectively. Also, other factors were shown to be statistically significant, including folate, Vit B12, HDL, TG, and height $(\mathrm{P}<0.05)$.

For females, the mean values of Hcy level for femaleCC, female-CT, female-TT were $8.85 \pm 3.53,9.22 \pm 3.34$, and $11.39 \pm 4.98 \mu \mathrm{mol}$, respectively. Also, a significant difference exists in the Hcy, Folate, Vit B12, G2h, and HDL between the female three genotype groups $(\mathrm{P}<0.05)$ (Table 1).

\section{The risk factors for HHcy in each sex and genotype}

In the males, after adjustment of weight, height, BMI, and other variables, we found that folate, VitB12, was negatively correlated with $\mathrm{HHcy}$, while $\mathrm{Cr}$ was positively correlated with HHcy. Also, genotype (CC and CT) was a protective factor for HHcy (Table S2). After stratified by genotypes, we found specific positive risk factors for each genotype, even though they have some common negative factors, including folate and VitB12. In the male CC group, age, $\mathrm{AST}$, and $\mathrm{Hb}$ were positive risk factors for HHcy; in the male CT group, age and AST are positive risk factors for HHcy; in the male TT group, SBP, AST, and $\mathrm{Hb}$ are positive risk factors for HHcy (Table 2).

In the females, we also found negative risk factors (genotype, folate, and VitB12) and positive risk factors (Cr) after adjustment of weight, height, BMI, and other variables (Table S2). We also found specific risk factors after stratifying by genotype, though they share the negative factors of folate and VitB12. In the female CT group, one positive risk factor $(\mathrm{Cr})$ was determined. In the female CT group, four positive risk factors (age, SBP, $\mathrm{Hb}$, and $\mathrm{Cr}$ ) are correlated with the Hcy level. In the female TT group, three positive risk factors (SBP, AST, and $\mathrm{Cr}$ ) were found to be correlated with Hcy concentration (Table 3).

\section{Discussion}

Plasma Hcy concentration is influenced by a diversity of physiologic and acquired factors and by interactions between such factors (6). People have found several factors that would cause HHcy, including MTHFR C677T polymorphism, folate, and vitamin B (27-29). An elevated level of the Hcy is connected with cardiovascular disease (CVD), stroke (30), and the neural tube defects, in addition to chronic renal failure (11), hepatocellular carcinoma (15), megaloblastic anemia, osteoporosis, depression, Alzheimer's disease, pregnancy problems, and others (5).

In the present study, we found that $\mathrm{Cr}$ was a common risk factor, which was positively correlated with Hcy concentration in all the groups. This positive relationship between $\mathrm{Cr}$ and Hcy was present in HHcy patients with or without hypertension $(31,32)$. Another evidence is that Hcy level could be increased in a dose-response effect by Guanidinoacetic acid (GAA), an intermediate in the 
Table 1 Clinical characteristics of the participants for each sex and genotype

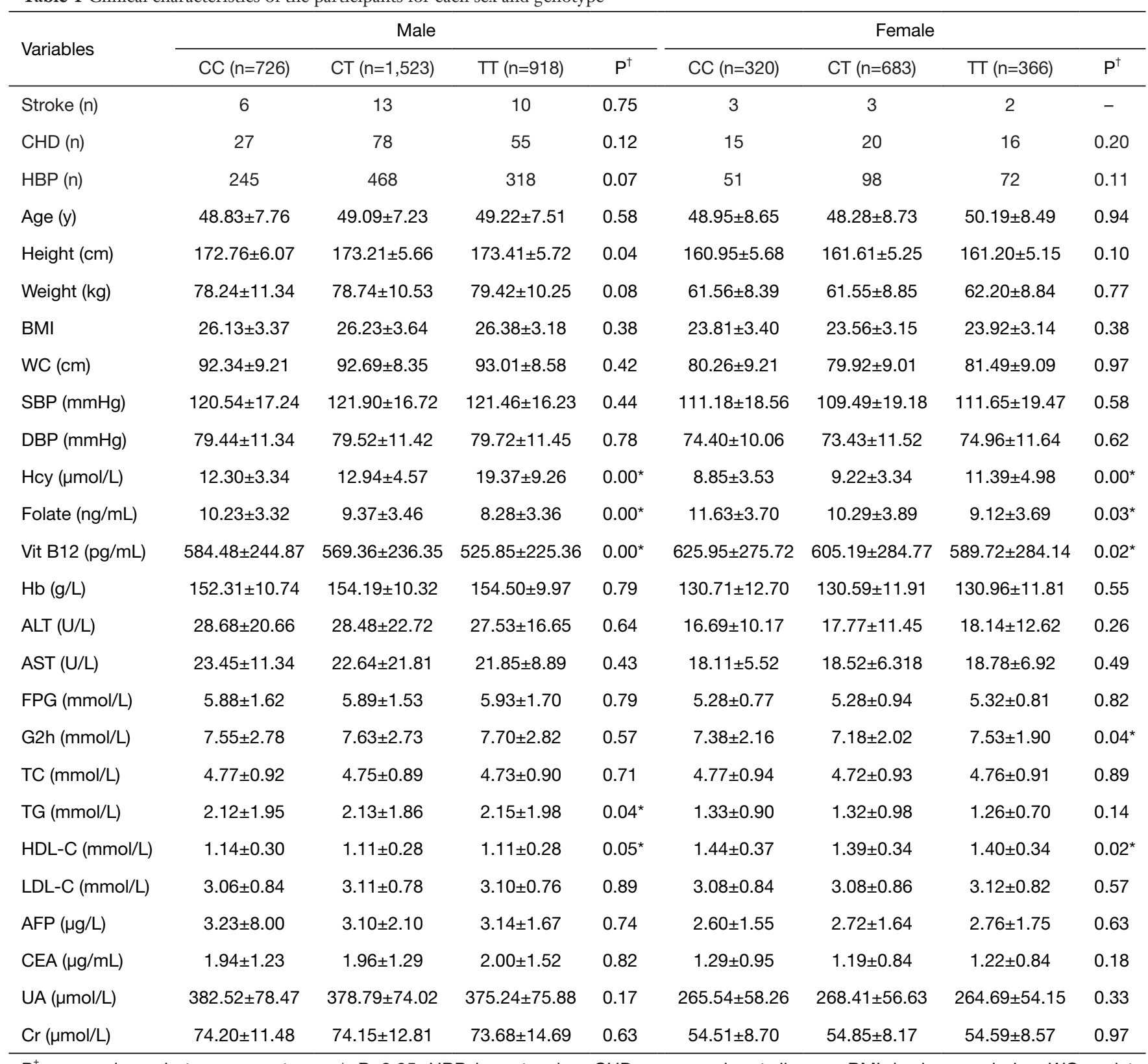

$\mathrm{P}^{\dagger}$, comparisons between genotypes; *, $\mathrm{P}<0.05$. HBP, hypertension; CHD, coronary heart disease; BMI, body mass index; WC, waist circumference; SBP, systolic blood pressure; DBP, diastolic blood pressure; Hcy, homocysteine; Hb, hemoglobin; FPG, fasting plasma glucose; G2h, postprandial 2 hours blood glucose; TC, total cholesterol; TG, triglyceride; CRP,C-reactive protein; ALT, alanine aminotransferase; AST, aspartate transaminase; LDL-C, low-density lipoprotein; HDL-C, high-density lipoprotein; AFP, alpha fetoprotein; CEA, carcinoembryonic antigen; UA, blood uric acid; Cr, blood creatinine. 
Table 2 Multilinear regression for the risk factors of HHcy for each genotype in males

\begin{tabular}{|c|c|c|c|c|c|}
\hline Sex-genotype & Variables & B & Standard error & Standardized coefficient & $P$ value \\
\hline \multirow{5}{*}{$\mathrm{M}-\mathrm{CC}$} & VitB12 & -0.00 & 0.00 & -0.21 & 0.00 \\
\hline & Age & 0.07 & 0.02 & 0.16 & 0.00 \\
\hline & AST & 0.05 & 0.02 & 0.20 & 0.00 \\
\hline & $\mathrm{Cr}$ & 0.05 & 0.01 & 0.17 & 0.00 \\
\hline & \multicolumn{5}{|c|}{ ANOVA: $F=25.37, P=0.00 ; R 2=0.25$, adjusted $R^{2}=0.24$} \\
\hline \multirow[t]{5}{*}{ M-CT } & (Constant) & 9.56 & 1.44 & & 0.00 \\
\hline & Folate & -0.41 & 0.03 & -0.31 & 0.00 \\
\hline & AST & 0.01 & 0.01 & 0.05 & 0.02 \\
\hline & $\mathrm{Cr}$ & 0.08 & 0.01 & 0.22 & 0.00 \\
\hline & \multicolumn{5}{|c|}{ ANOVA: $F=51.01, P=0.00 ; R 2=0.25$, adjusted $R^{2}=0.25$} \\
\hline \multirow[t]{6}{*}{ M-TT } & (Constant) & 10.35 & 5.13 & & 0.04 \\
\hline & Folate & -0.99 & 0.08 & -0.38 & 0.00 \\
\hline & VitB12 & -0.01 & 0.00 & -0.27 & 0.00 \\
\hline & SBP & 0.04 & 0.02 & 0.07 & 0.02 \\
\hline & $\mathrm{Hb}$ & 0.09 & 0.03 & 0.09 & 0.00 \\
\hline & \multicolumn{5}{|c|}{ ANOVA: $F=53.87, P=0.00 ; R 2=0.35$, adjusted $R^{2}=0.35$} \\
\hline
\end{tabular}

biosynthesis of $\mathrm{Cr}$ (33). It is documented that Hcy level increased in chronic renal failure (CRF), but the cause of HHcy is still unclear (34). However, the possibilities include decreased glomerular filtrate rate (GFR), defective renal or extrarenal metabolism (34). As a consequence of HHcy in renal failure, protein and DNA methylation are impaired, with an alteration in the allelic expression of genes regulated through methylation (35).

Aging was a contributing factor for CC and CT genotypes, not for TT genotype. This is unique from the other previous studies, which targets the whole genotype population $(25,31)$. We observed that participants with TT genotype had a peak level of Hcy at the early age of $20-40 \mathrm{y}$, with slightly change in the later years (Figure 1). The interpretation could be that the activity of the enzyme had been mostly lost in TT genotype at an early age, while the function was gradually damaged with aging by the nutritional and health status in the other two groups. Therefore, the intervention of decreasing Hcy should be performed at an early age for the TT genotype, while it could be intervened later for CC and CT genotypes.

SBP was positively correlated with HHcy in TT and CT genotypes. Previous studies have demonstrated that both SBP and HHcy are independent predictors for stroke morbidity in the hypertension population (36). However, the strength of their relationship was quite different from various studies. Data from the National Health and Nutrition Examination Survey (NHANES) showed that the positive relationship between Hcy and SBP was stronger in women than in men (37). However, it just showed a weak 
Table 3 Multilinear regression analysis for the risk factors of HHcy for each genotype in females

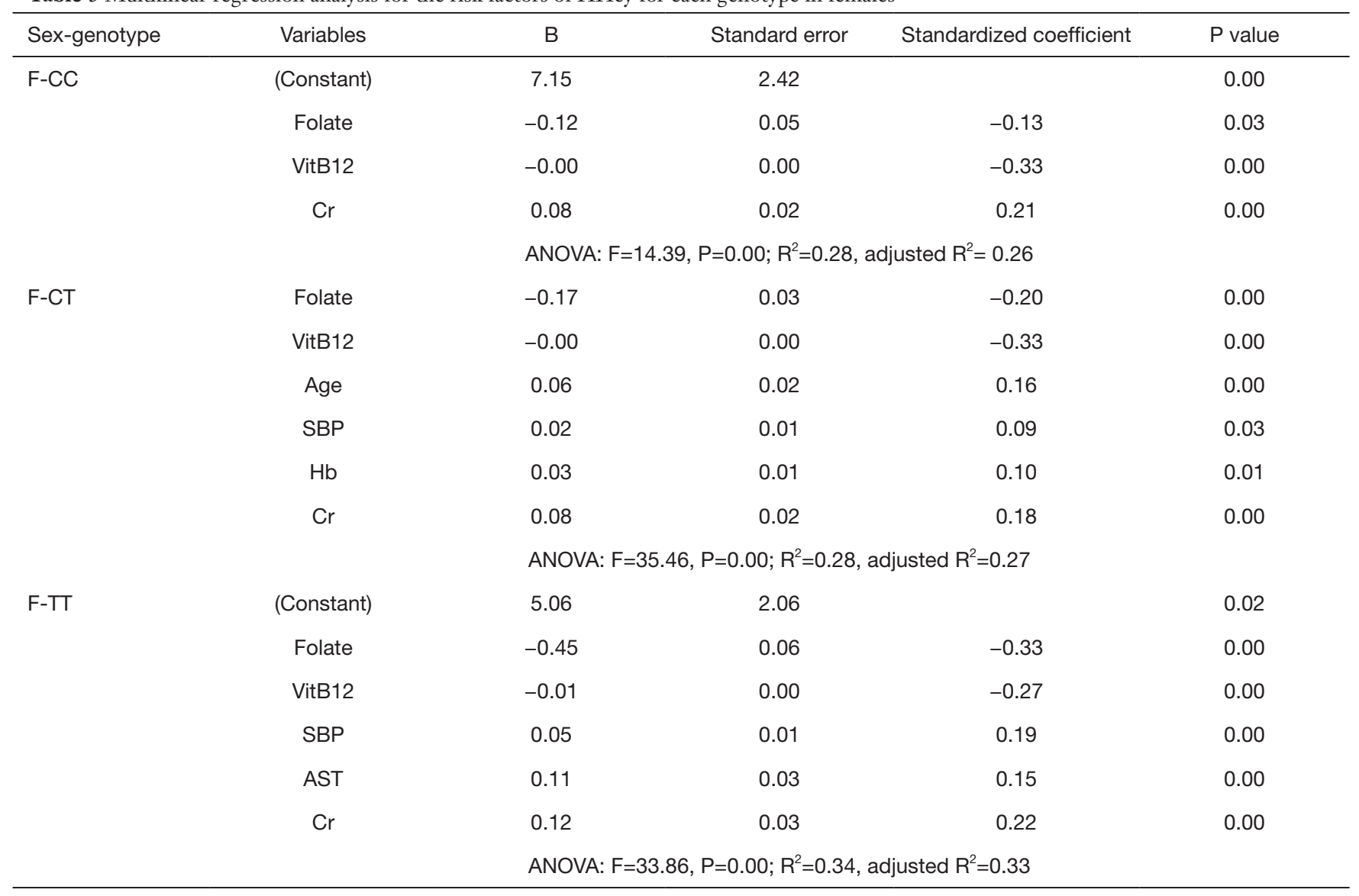
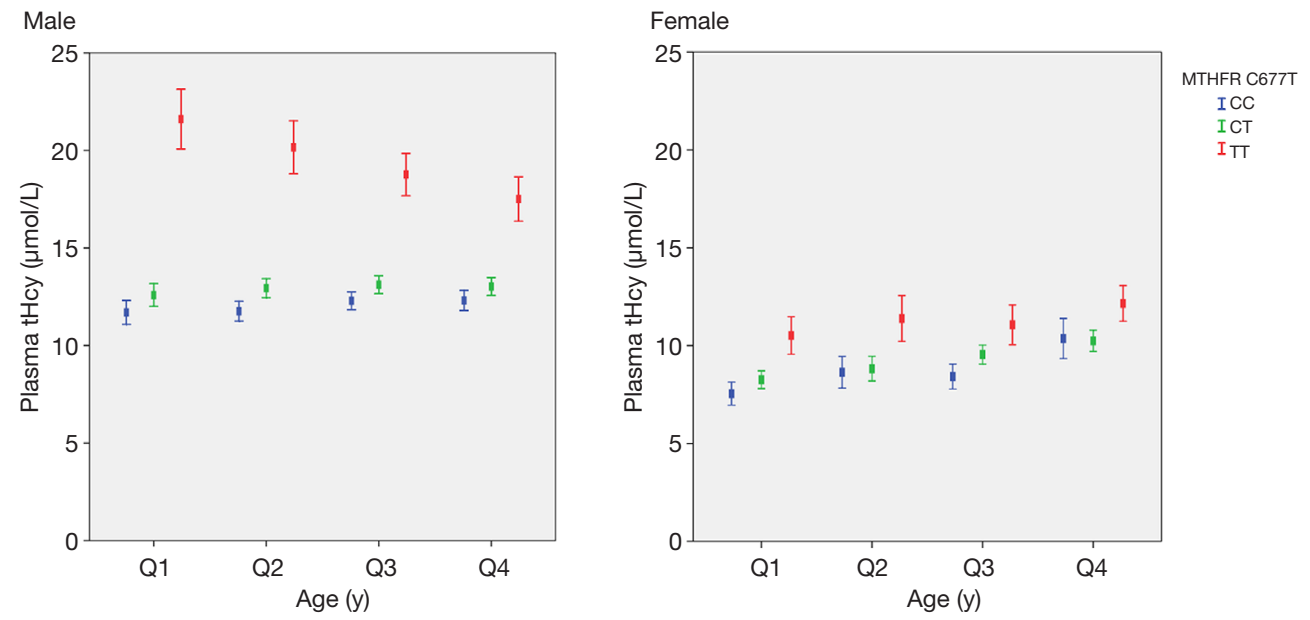

Figure 1 The correlation between age and the Hcy for each sex and genotype. 
Table 4 Risk factors for HHcy for each sex and MTHFR C677T genotype*

\begin{tabular}{lcccccc}
\hline Variables & F-CC & F-CT & F-TT & M-CC & M-CT & M-TT \\
\hline Folate & -0.12 & -0.17 & -0.45 & -0.27 & -0.41 & -0.99 \\
VitB12 & -0.00 & -0.00 & -0.01 & -0.00 & -0.01 & -0.01 \\
Cr & 0.08 & 0.08 & 0.12 & 0.05 & 0.08 & 0.11 \\
Age & - & 0.06 & - & 0.07 & 0.04 & - \\
SBP & - & 0.02 & 0.05 & - & - & 0.04 \\
AST & - & - & 0.11 & 0.05 & 0.01 & 0.09 \\
Hb & - & 0.03 & - & 0.03 & - & 0.09 \\
\hline
\end{tabular}

*, all these risk factors are significantly associated with HHcy levels $(P<0.05)$.

positive between Hcy and SBP in the Hordaland study (38). In the present study, we discovered that SBP was positively correlated with Hcy in TT and CT genotypes, but not in CC groups for both sexes (Table 4). This can partially explain the inconsistence of the studies. And it also suggested that HHcy with TT and CT genotypes are more prone to have H-type hypertension. However, it does not mean that HHcy would not influence SBP for the CC group, only showing that some other risk factors might outweigh this relationship.

AST is a positively correlated with Hcy for males (CC, CT, and TT) and female (TT). AST is an important index for hepatic function, and its concentration will rise when liver is damaged. Studies have shown that the plasma Hcy concentrations were significantly higher in patients with nonalcoholic fatty liver disease (39), while the Hcy concentrations were not affected by chronic viral hepatitis (40). However, the association between Hcy and AST was still controversial. Li et al. (41) did not find a significant correlation between Hcy and AST when they tried to investigate the effect of MTHFR gene polymorphisms and serum Hcy and folate levels on the hepatic functions in a hypertensive population. On the contrary, Huang et al. found an inverse relationship between Hcy and AST in hemodialysis patients, whose remethylation of Hcy was impaired (42). The nonconformity of these studies was probably from the selection criteria of the subject.

We also found a positive correlation between $\mathrm{Hb}$ and HHcy in males (CC and TT) and females (CT). At present, few studies have focused on the association between Hcy and $\mathrm{Hb}$, without uniform conclusion. A positive relationship has been reported in a group of patients with CVD (43). However, a negative relationship was presented in Behcet's disease and atrophic glossitis, which might be result from nutrition deficiency. It is still unclear for this positive relationship between $\mathrm{Hb}$ and $\mathrm{HHcy}$, and further studies are needed to explore its mechanism.

The strength of this study is that we classify people into separate sex and genotype groups, both of which are strong independent variables for HHcy. There are several benefits to this classification. First, we exclude some confound bias among groups, including age, which shows different influential strength among groups. Second, we discovered some new variables that may influence the Hcy level, even though they still need further prospective studies to confirm.

However, there are still some limitations to this study. First, there might be more risk factors to be discovered in addition to our findings. The variable selected for this study was only the physical and laboratory examination. Other factors should be taken into consideration as they were proved to be essential for Hcy metabolism, including smoking, drinking, nutrition, physical exercise, etc. Second, the correlation between HHcy and the risk factors in this cross-section study cannot reflect the causal relationship. A well-designed cohort study or randomized clinical trial is needed to confirm the causality. Third, the result of this single-center study would be corroborated by multi-center collaborations, and it would be more authentic if the result can be verified in different centers in the future.

In summary, we have found that except the common protective factors (folate and Vit B12) and risk factor (Cr), each sex and genotype group has its specific risk factors for HHcy: female-CT (age, SBP, and Hb), female-TT (SBP and AST), male-CC (age, AST and Hb), male-CT (age and AST) and male-TT (SBP, AST, and $\mathrm{Hb}$ ). These results might be useful for predicting or prevention of systemic diseases. 


\section{Acknowledgments}

Funding: The present study was supported by the National Natural Science Foundation of China (No. 81870249) and the National Natural Science Foundation of China International Cooperation program (No. 81561128020).

\section{Footnote}

Reporting Checklist: The authors have completed the MDAR reporting checklist. Available at http://dx.doi.org/10.21037/ atm-20-6587

Data Sharing Statement: Available at http://dx.doi. org/10.21037/atm-20-6587

Conflicts of Interest: All authors have completed the ICMJE uniform disclosure form (available at http://dx.doi. org/10.21037/atm-20-6587). The authors have no conflicts of interest to declare.

Ethical Statement: The authors are accountable for all aspects of the work in ensuring that questions related to the accuracy or integrity of any part of the work are appropriately investigated and resolved. The Ethics Committee at PLA General Hospital approved the protocol of this cross-sectional, observational, non-randomized study (clinical trial ID: S2016-098-02), which was designed following the principle of the Declaration of Helsinki (as revised in 2013). All subjects gave their informed consent to take part in the study. Written informed consent was obtained from all patients to publish their information in nominate data.

Open Access Statement: This is an Open Access article distributed in accordance with the Creative Commons Attribution-NonCommercial-NoDerivs 4.0 International License (CC BY-NC-ND 4.0), which permits the noncommercial replication and distribution of the article with the strict proviso that no changes or edits are made and the original work is properly cited (including links to both the formal publication through the relevant DOI and the license). See: https://creativecommons.org/licenses/by-nc-nd/4.0/.

\section{References}

1. Zaric BL, Obradovic M, Bajic V, et al. Homocysteine and Hyperhomocysteinaemia. Curr Med Chem
2019;26:2948-61.

2. Finkelstein JD. Methionine metabolism in mammals. J Nutr Biochem 1990;1:228-37.

3. Taguchi T, Mori H, Hamada A, et al. Serum folate, total homocysteine levels and methylenetetrahydrofolate reductase $677 \mathrm{C}>\mathrm{T}$ polymorphism in young healthy female Japanese. Asia Pac J Clin Nutr 2012;21:291-5.

4. Zappacosta B, Graziano M, Persichilli S, et al. 5,10-Methylenetetrahydrofolate reductase (MTHFR) C677T and A1298C polymorphisms: genotype frequency and association with homocysteine and folate levels in middle-southern Italian adults. Cell Biochem Funct 2014;32:1-4.

5. Liew SC, Gupta ED. Methylenetetrahydrofolate reductase (MTHFR) C677T polymorphism: epidemiology, metabolism and the associated diseases. Eur J Med Genet 2015;58:1-10.

6. Zeng Q, Li F, Xiang T, et al. Influence of food groups on plasma total homocysteine for specific MTHFR C677T genotypes in Chinese population. Mol Nutr Food Res 2017;61:4.

7. Bjørke Monsen AL, Ueland PM. Homocysteine and methylmalonic acid in diagnosis and risk assessment from infancy to adolescence. Am J Clin Nutr 2003;78:7-21.

8. Ueland PM, Refsum H. Plasma homocysteine, a risk factor for vascular disease: plasma levels in health, disease, and drug therapy. J Lab Clin Med 1989;114:473-501.

9. Homocysteine Studies Collaboration. Homocysteine and risk of ischemic heart disease and stroke: a meta-analysis. JAMA 2002;288:2015-22.

10. Yan L, Zhao L, Long Y, et al. Association of the maternal MTHFR C677T polymorphism with susceptibility to neural tube defects in offsprings: evidence from 25 casecontrol studies. PLoS One 2012;7:e41689.

11. Canepa A, Carrea A, Caridi G, et al. Homocysteine, folate, vitamin B12 levels, and C677T MTHFR mutation in children with renal failure. Pediatr Nephrol 2003;18:225-9.

12. Jiang $Y$, Xia $X$, Wang $W$, et al. Hyperhomocysteinemia and related genetic polymorphisms correlate with ulcerative colitis in Chinese Han population in Central China (corrected). Cell Biochem Biophys 2012;62:203-10.

13. Chen XL, Wang YM, Zhao F, et al.

Methylenetetrahydrofolate reductase polymorphisms and colorectal cancer prognosis: A meta-analysis. J Gene Med 2019;21:e3114.

14. Gohari M, Dastgheib SA, Jafari-Nedooshan J, et al. Association of MTHFR 677C\&gt;T, 1298A\&gt;C 
and MTR 2756A\&gt;G Polymorphisms with Risk of Retinoblastoma. Klin Onkol 2019;32:375-9.

15. Qi YH, Yao LP, Cui GB, et al. Meta-analysis of MTHFR C677T and A1298C gene polymorphisms: association with the risk of hepatocellular carcinoma. Clin Res Hepatol Gastroenterol 2014;38:172-80.

16. Li WX, Lv WW, Dai SX, et al. Joint associations of folate, homocysteine and MTHFR, MTR and MTRR gene polymorphisms with dyslipidemia in a Chinese hypertensive population: a cross-sectional study. Lipids Health Dis 2015;14:015-0099.

17. Shi TL, Wu Y, Li Y, et al. The relevance of MTHFR C677T, A1298C, and MTRR A66G polymorphisms with response to male infertility in Asians: A meta-analysis. Medicine 2019;98:e14283

18. Lin Z, Li Q, Sun Y, et al. Interactions between genetic variants involved in the folate metabolic pathway and serum lipid, homocysteine levels on the risk of recurrent spontaneous abortion. Lipids Health Dis 2019;18:143.

19. Rainero I, Vacca A, Roveta F, et al. Targeting MTHFR for the treatment of migraines. Expert Opin Ther Targets 2019;23:29-37.

20. Wang BR, Ou Z, Jiang T, et al. Independent Correlation of Serum Homocysteine with Cerebral Microbleeds in Patients with Acute Ischemic Stroke due to Large-Artery Atherosclerosis. J Stroke Cerebrovasc Dis 2016;25:2746-51.

21. Bates CJ, Mansoor MA, Gregory J, et al. Correlates of plasma homocysteine, cysteine and cysteinyl-glycine in respondents in the British National Diet and Nutrition Survey of young people aged 4-18 years, and a comparison with the survey of people aged 65 years and over. Br J Nutr 2002;87:71-9.

22. Refsum H, Ueland PM, Svardal AM. Fully automated fluorescence assay for determining total homocysteine in plasma. Clin Chem 1989;35:1921-7.

23. Fiskerstrand T, Refsum H, Kvalheim G, et al. Homocysteine and other thiols in plasma and urine: automated determination and sample stability. Clin Chem 1993;39:263-71.

24. Zironi E, Gazzotti T, Barbarossa A, et al. Technical note: development and validation of a method using ultra performance liquid chromatography coupled with tandem mass spectrometry for determination of vitamin B12 concentrations in milk and dairy products. J Dairy Sci 2013;96:2832-6.

25. Wang Y, Li X, Qin X, et al. Prevalence of hyperhomocysteinaemia and its major determinants in rural Chinese hypertensive patients aged $45-75$ years. The
British journal of nutrition 2013;109:1284-93.

26. Ventura P, Panini R, Verlato C, et al.

Hyperhomocysteinemia and related factors in 600

hospitalized elderly subjects. Metabolism: clinical and experimental 2001;50:1466-71.

27. Fekih-Mrissa N, Mrad M, Klai S, et al.

Methylenetetrahydrofolate reductase (C677T and

A1298C) polymorphisms, hyperhomocysteinemia, and ischemic stroke in Tunisian patients. J Stroke Cerebrovasc Dis 2013;22:465-9.

28. Hustad S, Midttun O, Schneede J, et al. The methylenetetra- hydrofolate reductase $677 \mathrm{C}-->\mathrm{T}$ polymorphism as a modulator of a $\mathrm{B}$ vitamin network with major effects on homocysteine metabolism. Am J Hum Genet 2007;80:846-55.

29. He JA, Hu XH, Fan YY, et al. Hyperhomocysteinaemia, low folate concentrations and methylene tetrahydrofolate reductase C677T mutation in acute mesenteric venous thrombosis. Eur J Vasc Endovasc Surg 2010;39:508-13.

30. Huh HJ, Chi HS, Shim EH, et al. Gene--nutrition interactions in coronary artery disease: correlation between the MTHFR C677T polymorphism and folate and homocysteine status in a Korean population. Thromb Res 2006;117:501-6.

31. Han L, Liu Y, Wang C, et al. Determinants of hyperhomocysteinemia in healthy and hypertensive subjects: A population-based study and systematic review. Clin Nutr 2017;36:1215-30.

32. Yang Q, Lu Y, Deng Y, et al. Homocysteine level is positively and independently associated with serum creatinine and urea nitrogen levels in old male patients with hypertension. Sci Rep 2020;10:18050.

33. Ostojic SM, Stojanovic M, Drid P, et al. Dose-response effects of oral guanidinoacetic acid on serum creatine, homocysteine and B vitamins levels. Eur J Nutr 2014;53:1637-43.

34. Satta E, Perna AF, Lombardi C, et al. Hyperhomocysteinemia in chronic renal failure. G Ital Nefrol 2006;23:480-9.

35. Perna AF, Ingrosso D, Satta E, et al. Homocysteine metabolism in renal failure. Curr Opin Clin Nutr Metab Care 2004;7:53-7.

36. Pang H, Han B, Fu Q, et al. Association of High Homocysteine Levels With the Risk Stratification in Hypertensive Patients at Risk of Stroke. Clin Ther 2016;38:1184-92.

37. Lim U, Cassano PA. Homocysteine and blood pressure in the Third National Health and Nutrition Examination 
Survey, 1988-1994. Am J Epidemiol 2002;156:1105-13.

38. Nygård O, Vollset SE, Refsum H, et al. Total plasma homocysteine and cardiovascular risk profile. The Hordaland Homocysteine Study. JAMA 1995;274:1526-33.

39. Dai Y, Zhu J, Meng D, et al. Association of homocysteine level with biopsy-proven non-alcoholic fatty liver disease: a meta-analysis. J Clin Biochem Nutr 2016;58:76-83.

40. Gulsen M, Yesilova Z, Bagci S, et al. Elevated plasma homocysteine concentrations as a predictor of steatohepatitis in patients with non-alcoholic fatty liver disease. J Gastroenterol Hepatol 2005; 20:1448-55.

41. Li WX, Li W, Cao JQ, et al. Folate Deficiency Was

Cite this article as: Xiang T, Xiang $\mathrm{H}$, Yan M, Yu S, Horwedel MJ, Li Y, Zeng Q. Systemic risk factors correlated with hyperhomocysteinemia for specific MTHFR C677T genotypes and sex in the Chinese population. Ann Transl Med 2020;8(21):1455. doi: 10.21037/atm-20-6587
Associated with Increased Alanine Aminotransferase and Glutamyl Transpeptidase Concentrations in a Chinese Hypertensive Population: A Cross-Sectional Study. J Nutr Sci Vitaminol (Tokyo) 2016;62:265-71.

42. Huang JW, Yen CJ, Pai MF, et al. Association between serum aspartate transaminase and homocysteine levels in hemodialysis patients. Am J Kidney Dis 2002;40:1195-201.

43. Schaffer A, Verdoia M, Cassetti E, et al. Relationship between homocysteine and coronary artery disease. Results from a large prospective cohort study. Thromb Res 2014;134:288-93. 